\title{
Como que as mudanças nas taxas de mortalidade e expectativa de vida afetam a projeção da população idosa no estado de São Paulo?'
}

\section{How do changes in mortality and life expectancy affect the elderly population projection in the state of Sao Paulo, Brazil?}

\author{
Gustavo Pedroso de Lima Brusse ${ }^{1}$ (D)
}

'Instituto de Filosofia e Ciências Humanas (IFCH), Nucleo de Estudos de População 'Elza Berquó' - NEPO, Universidade Estadual de Campinas (UNICAMP) - Campinas (SP), Brasil.

Como citar: Brusse GPL. Como que as mudanças nas taxas de mortalidade e expectativa de vida afetam a projeção da população idosa no estado de São Paulo?. Cad Saúde Colet, 2021;29(esp.):144-151. https://doi.org/10.1590/1414$462 \times 202199010422$

\section{Resumo}

Introdução: O Brasil experimentou, ao longo do século XX, uma profunda mudança em relação à expectativa de vida ao nascer. Nesse processo, foi importante não somente a queda do nível da mortalidade para o grande aumento da expectativa de vida, mas também a mudança no padrão da mortalidade. Recentemente, vêm sendo discutidas mudanças no padrão de mortalidade nas idades mais avançadas, bem como o impacto no envelhecimento populacional. Entretanto, no Brasil, as discussões sobre a longevidade e o comportamento da mortalidade nas idades avançadas ainda são incipientes. Especialistas sugerem que projeções de mortalidade incorporem novas tendências de desaceleração da mortalidade em idades avançadas e explorem abordagens de coortes para suas formulações de tendências. Objetivo: Estudar o efeito causado pelas mudanças no comportamento da mortalidade e da expectativa de vida na predição do tamanho da população idosa do estado de São Paulo. Método: Método estendido de coortes componentes. Resultados: Alterações no nível e no padrão da mortalidade têm um efeito maior na predição da população de 80 anos ou mais em comparação com a população de 60 anos ou mais. Ademais, considerar o gap da expectativa de vida entre os sexos é relevante para determinar o número futuro de idosos. Conclusão: Ao utilizar países em diferentes estágios da transição epidemiológica como cenário futuro do padrão e do nível da mortalidade de São Paulo, o método estendido de coortes componentes se torna uma possibilidade metodológica interessante para avaliar o impacto dessas modificações para a projeção da população idosa, podendo ser uma ferramenta para a avaliação de políticas públicas.

Palavras-chave: projeção populacional; população idosa; envelhecimento populacional; expectativa de vida; mortalidade.

\begin{abstract}
Background: Brazil experienced a significant change in life expectancy at birth in the 20th century. In this process, it is important to observe not only the decrease in mortality level and the increase in life expectancy, but also the change in mortality pattern, that is, how mortality occurred and which ages were most impacted. Recently, these changes in the mortality pattern at more advanced ages and their
\end{abstract}

'Artigo submetido Chamada Pública para Número Temático da Revista Cadernos Saúde Coletiva "Demografia e Saúde" para o eixo: Debate sobre curso de vida e lifespan.

Trabalho realizado na Universidade Estadual de Campinas (UNICAMP) - Campinas (SP), Brasil

Correspondência: Gustavo Pedroso de Lima Brusse. E-mail: gustavo.brusse@gmail.com

Fonte de financiamento: CAPES.

Conflito de interesses: nada a declarar.

Recebido em: Nov. 11, 2019. Aprovado em: Mar. 15, 2020
Este é um artigo publicado em acesso aberto (Open Access) sob a licença Creative Commons Attribution, que permite uso, distribuição e reprodução em qualquer meio, sem restrições desde que o trabalho original seja corretamente citado. 
impact on population aging have been discussed more intensively. However, in Brazil, discussions about human longevity and mortality behavior in advanced ages are still incipient. Experts suggest that future studies and mortality projections incorporate new trends in decelerating mortality at advanced ages and explore cohort approaches in their trend formulations. Objective: To study the effect of changes in mortality and life expectancy on predicting the elderly population size in the state of Sao Paulo, Brazil. Method: Extended cohort-component study. Results: Changes in the level and pattern of mortality have a greater effect on the population aged $\geq 80$ compared with that on the population aged $\geq 60$. In addition, considering the life expectancy gap between sexes is relevant to determine the future number of older people. Conclusion: The extended cohort component method is an interesting methodological approach to assess the impact of mortality changes on the elderly population projection by using different stages of the epidemiological transition as a future scenario

Keywords: population projection; elderly population; population aging; life expectancy; mortality.

\section{INTRODUÇÃOO}

A mudança entre distintos regimes de mortalidade é chamada de transição epidemiológica, processo que tem ocorrido durante um longo período nos países desenvolvidos. Omrnan ${ }^{1}$ define a transição epidemiológica como um conjunto de mudanças no padrão da mortalidade e morbidade. Nesse mesmo sentido, Horiuchi ${ }^{2}$ a define como um processo que pode ser descrito por meio de cinco transições: em um primeiro momento, as mortes seriam causadas, majoritariamente, por fatores externos e passariam a ser causadas por doenças infecciosas; em um segundo momento, passariam de uma maior prevalência de doenças infecciosas para doenças degenerativas; em um terceiro momento, haveria um declínio das doenças cardiovasculares; em um quarto momento, ocorreria um declínio de mortes causadas por câncer; e na última etapa da transição, haveria uma queda da senescência.

Esse processo tem ocorrido de forma desigual e em ritmos diferenciados, principalmente entre os países em desenvolvimento. Na América Latina e no Caribe, Frenk et al. ${ }^{3}$ destacam a maior complexidade desse fenômeno, pois há uma sustentada, porém insuficiente, queda dos óbitos causados por doenças infecciosas e parasitárias, ao mesmo tempo que há um rápido crescimento de processos crônicos, ocasionando uma superposição de etapas. Durante o século XX, esses países experimentaram uma profunda mudança em relação à expectativa de vida ao nascer. Entretanto, não somente foi importante a queda do nível da mortalidade para o grande aumento da expectativa de vida, mas também a mudança no padrão da mortalidade, ou seja, de que forma ela ocorre e em quais idades possui maior impacto.

Nas idades mais avançadas, muitos demógrafos e gerontologistas acreditavam que taxas de mortalidade não poderiam ser mais reduzidas em razão de causas naturais e processos de senescência ${ }^{4}$. Mas o que ocorre nos países em desenvolvimento é um sustentado processo de redução das taxas de mortalidade, que chega até $1 \%$ ao ano e resulta em uma longevidade e em um aumento da proporção de idosos nunca visto antes ${ }^{4}$. $O$ aumento da expectativa de vida pode chegar a 1 ano por década, e, se a redução nas taxas de mortalidade for de $2 \%$, poderá ser de 2 anos por década 5 . Segundo uma projeção populacional dos Estados Unidos, a população acima de 85 anos pode aumentar para 70 milhões em 2080 , com $2 \%$ de redução da mortalidade idosa a cada ano $0^{6,7}$.

Entretanto, o conhecimento acerca dos determinantes da longevidade humana ainda é considerado muito vago, sendo que apenas poucos avanços foram obtidos nessa área nos últimos anos. Autores como Christensen e Vaupe ${ }^{8}$, Crimmins ${ }^{9}$ e Passarino e Rangod ${ }^{10}$ tentam desvendar os determinantes da longevidade humana entre fatores médicos, genéticos, ambientais e comportamentais. Mas, diferentemente de outras idades, ainda não se sabe quais desses fatores teriam a maior influência na queda das taxas de mortalidade nas idades avançadas. Estudos recentes usam a abordagem de ciclo de vida, argumentando que a queda das taxas de mortalidade nas idades avançadas seria consequência da trajetória de vida dos indivíduos, como a maior escolaridade, a menor exposição a riscos de morte e até mesmo as condições de nascimento e da infância do indivíduo ${ }^{8}$. Essa maior sobrevivência da população nas primeiras idades e nas idades jovens e adultas, assim como a concentração de mortes cada vez em idades mais avançadas, pode ser percebida pela "horizontalização" da curva de 
sobrevivência e pelo aumento da idade média de morte ${ }^{11}$, também conhecido como processo de compressão da mortalidade ${ }^{12,13}$.

Além da queda das taxas de mortalidade e do aumento da expectativa de vida, há um consenso acerca da existência de diferenças entre os sexos. Desde o século XVIII, quando foram construídas as primeiras tábuas de vida, já se conhecia a existência de diferenças entre os sexos quanto à expectativa de vida. Entretanto, até os dias atuais, não se sabe ao certo o que motiva essa diferença. Além disso, o padrão diferenciado de mortalidade entre homens e mulheres gera profundos questionamentos e conclusões paradoxais. Por exemplo, Luy e Minigawa 14:12 descrevem que: "[...] mulheres ficam mais doentes, mas homens morrem mais rapidamente [...]", e que "[...] os homens possuem taxas de mortalidade maiores do que as mulheres em quase todas as idades, mas mulheres relatam ter saúde pior que os homens". As sugestões para explicar essa diferença entre os sexos incluem os fatores biológicos (genéticos e hormonais), riscos adquiridos por causa de papéis sociais, condições de ambiente e de comportamento. Pelo fato que a mortalidade dos homens, principalmente nas idades jovens e adultas, difere significativamente do padrão encontrado para as mulheres, alguns autores concluem que os riscos adquiridos por meio de papéis sociais são os que mais explicam as diferenças entre a longevidade dos homens e das mulheres ${ }^{14}$. Essa conclusão é corroborada por estudos que demostram que, em grupos em que homens e mulheres possuem socialmente um estilo de vida similar, as diferenças da expectativa de vida por sexo tendem a ser menores ${ }^{15}$.

No Brasil, de Moura et al. ${ }^{16: 779}$ concluíram que a"[...] razão de mortalidade por causas externas chega a ser quase nove vezes maior entre homens comparativamente às mulheres, com valores maiores nas regiões Norte e Nordeste", e que essa diferença pode ser explicada pela abordagem de gênero, ao verificar que "[...] os homens se expõem mais a situações de acidentes e violência por conta de comportamentos reafirmadores da masculinidade"16:783. Os principais tipos de óbitos por causas externas identificados entre homens foram as agressões, seguidas por acidentes de transporte terrestre - o inverso das mulheres. Além do sexo, a idade foi o fator preditivo mais importante da mortalidade precoce por causas externas, evidenciando, conforme a literatura, que o grande diferencial entre os sexos realmente são os homens nas idades jovens.

Utilizando o método estendido de coortes componentes para a projeção da população idosa, desenvolvido por Zeng et al. ${ }^{17}$, o objetivo deste trabalho consistiu em estudar o efeito causado pelas mudanças no comportamento da mortalidade e da expectativa de vida na predição do tamanho da população idosa do estado de São Paulo, tendo em vista os principais pontos destacados pela literatura: redução nas taxas de mortalidade nas idades avançadas e manutenção das diferenças da expectativa de vida entre os sexos.

\section{MÉTODO}

No Brasil, as fontes de dados mais utilizadas para se obter a informação sobre a quantidade de óbitos na população são o registro civil, o Sistema de Informação sobre a Mortalidade (SIM) e o Censo Demográfico. Nas duas primeiras fontes de dados, a unidade de enumeração é o evento demográfico, diferentemente do Censo, cuja unidade é o indivíduo, ou seja, a informação sobre os óbitos depende da declaração do entrevistado. Enquanto em muitas regiões o registro civil e as estatísticas vitais podem não ter uma cobertura satisfatória do registro de óbitos por causa da falta de abrangência dos sistemas, o Censo pode apresentar diversos problemas de subenumeração dos óbitos por depender da declaração dos entrevistados.

Queiroz e Sawyer ${ }^{18}$ fizeram uma comparação da informação obtida pelo quesito "óbitos ocorridos no domicílio nos últimos 12 meses", coletada pelo Censo Demográfico em 2010, com as estimativas produzidas com os dados de mortalidade do SIM. Os autores citaram diversas limitações do Censo, como possibilidade de dissolução do domicílio após a morte de um membro, de pessoas poderem ser consideradas membros de mais de um domicílio, erros na declaração do período de referência e na declaração da idade tanto dos vivos como dos mortos. Além disso, Paes ${ }^{19}$ concluiu que a cobertura dos óbitos utilizando os dados do SIM foi classificada, no mínimo, como "boa" para todos os estados do Sul, Sudeste, Centro-Oeste e parte do Nordeste. Isso compreende que, nessas regiões, a cobertura atingiu um nível igual ou 
superior a 90\%. Em São Paulo, a cobertura estimada foi de $94,3 \%$ para os homens e de $96,2 \%$ para as mulheres. Além disso, o autor apontou que o número de óbitos registrados pelo SIM foi maior do que o registrado pelo registro civil para quase todas as idades, mas as diferenças absolutas entre os resultados produzidos pelas duas fontes de dados “[...] não foram suficientes para produzir padrões de mortalidade diferenciados[..."19:883. Apesar de os dados do SIM no estado de São Paulo serem de boa cobertura, chama atenção o fato de que todos os métodos utilizados por Paes ${ }^{19}$ não empregam as idades mais avançadas para estimar a cobertura dos óbitos; portanto, não se pode ter certeza da qualidade e da cobertura da mortalidade nessas idades, o que demanda estudos futuros nessa área.

Tendo em vista as desvantagens da utilização do Censo como fonte de dados para a mortalidade, como a não presença do quesito "óbitos ocorridos no domicílio nos últimos 12 meses" em 2000 e os fortes pressupostos para a aplicação de métodos para a correção do nível da mortalidade, este trabalho seguiu o estudo de Paes ${ }^{19}$, considerando o SIM como uma fonte de dados segura para estimar a mortalidade do estado de São Paulo. Para a aplicação do método estendido de coortes componentes, é necessária a informação sobre as probabilidades de sobrevivência por idades simples e esperança de vida ao nascer. Foram utilizados os microdados do SIM disponibilizados pelo DATASUS. As taxas específicas de mortalidade por idade simples foram calculadas por meio da média dos óbitos do período de três anos - 1999, 2000 e 2001 para o cálculo dos óbitos de 2000, e 2009, 2010 e 2011 para o cálculo dos óbitos de 2010 -, de forma a evitar que as taxas de mortalidade fossem influenciadas pela flutuação do número de óbitos de um único ano. A partir dessas taxas, foram construídas as tábuas de mortalidades completas para cada sexo no estado de São Paulo em 2000 e 2010. As esperanças de vida ao nascer e aos 60 anos são apresentadas na Tabela 1 e comparadas com as calculadas pelo IBGE/DPE20.

Tabela 1. Comparação das esperanças de vida ao nascer e aos 60 anos, calculada com dados do SIM e divulgadas pelo IBGE/DPE, estado de São Paulo, 2000 e 2010

\begin{tabular}{|c|c|c|c|c|}
\hline \multirow{2}{*}{ Esperança de vida / sexo } & \multicolumn{2}{|c|}{2000} & \multicolumn{2}{|c|}{2010} \\
\hline & Calculada & IBGE/DPE & Calculada & IBGE/DPE \\
\hline \multicolumn{5}{|l|}{ Mulheres } \\
\hline Esperança de vida ao nascer & 76,9 & 76,7 & 79,3 & 79,3 \\
\hline Esperança de vida aos 60 anos & 22,3 & 21,5 & 23,6 & 23,7 \\
\hline \multicolumn{5}{|l|}{ Homens } \\
\hline Esperança de vida ao nascer & 68,1 & 67,0 & 72,3 & 72,6 \\
\hline Esperança de vida aos 60 anos & 18,5 & 17,5 & 19,9 & 19,8 \\
\hline
\end{tabular}

Fonte: elaborada a partir dos dados do SIM/DATASUS ${ }^{25}$ e IBGE/DPE ${ }^{20}$

\section{O MÉTODO ESTENDIDO DE COORTES COMPONENTES}

O método estendido de coortes componentes, desenvolvido por Zeng ${ }^{17}$ Zeng et al. ${ }^{21}$, é um modelo de macrossimulação fundamentado nas tábuas de vida de Bongaarts ${ }^{22}$ e Zeng ${ }^{17}$, que possibilita a projeção da população segundo a idade, o sexo, o estado conjugal, entre outras variáveis demográficas ${ }^{21}$. O método utiliza taxas de transição entre os diversos estados demográficos que os indivíduos experienciam durante suas respectivas trajetórias de vida e foi pensado, inicialmente, para a projeção de arranjos domiciliares. Neste trabalho, foi utilizado o método estendido de coortes componentes para estudar o efeito causado pelas mudanças no comportamento da mortalidade e da expectativa de vida na predição do tamanho da população idosa de 65 anos ou mais e 85 anos ou mais. Foram comparados os resultados das projeções realizadas com três diferentes cenários em relação à expectativa de vida e diferentes cenários em relação ao nível e padrão da mortalidade. Foi assumido que as taxas de fecundidade e migração permaneceram constantes para o estado de São Paulo nos níveis de 2010 para todo o período de projeção. 


\section{CENÁRIOS DE MORTALIDADE PARA O ESTADO DE SÃO PAULO}

Usando a interpolação linear da função Logito de sobrevivência ${ }^{23}$ entre uma tabela de vida inicial (estado de São Paulo em 2010) e uma tabela de limite de vida final, foram criados diferentes cenários para a evolução das taxas de mortalidade específicas por idade e expectativa de vida ao nascer (Figura 1). Para isso, foram escolhidos países que integram o projeto Human Mortality Database (HMD) a fim de representar padrões futuros de altos níveis de mortalidade e baixos níveis de mortalidade. Analisando o padrão de mortalidade desses países, a Rússia foi escolhida para representar um padrão de alta mortalidade de adultos (principalmente entre os homens) e idosos, enquanto o Japão foi escolhido para representar um padrão de baixa mortalidade de adultos e idosos.

- Cenário 1: alta mortalidade

Este cenário testou qual seria o impacto no número de idosos caso a expectativa de vida e as taxas específicas de mortalidade por idade do estado de São Paulo convergissem para o padrão e nível da Rússia (2014) durante o período de 2010 a 2050. Foram interpoladas as taxas específicas de mortalidade por idade de São Paulo pelo padrão russo, assumindo, portanto, a hipótese de aumento das taxas de mortalidade adulta, principalmente masculina, e hipótese de aumento da mortalidade em idades mais avançadas.

\section{- Cenário 2: taxas constantes}

Este cenário testou qual seria o impacto no número de idosos no estado de São Paulo se a expectativa de vida e as taxas específicas de mortalidade por idade se mantivessem constantes durante o período de 2010 a 2050.

\section{- Cenário 3: baixa mortalidade}

Este cenário testou qual seria o impacto no número de idosos no estado de São Paulo caso a expectativa de vida e as taxas específicas de mortalidade por idade do Estado de São Paulo convergissem para o padrão e nível do Japão (2010) durante o período de 2010 a 2050. Foram interpoladas as taxas específicas de mortalidade por idade de São Paulo pelo padrão japonês, assumindo, portanto, a hipótese de diminuição das taxas de mortalidade adulta e a hipótese de diminuição da mortalidade nas idades avançadas.

Para testar o impacto do gap entre as expectativas de vidas entre homens e mulheres, partiu-se do cenário de baixa mortalidade (cenário 3), e mais três hipóteses foram criadas:

- Cenário 3.1: hipótese de 9 anos de diferença entre a expectativa de vida das mulheres e dos homens (manutenção das diferenças da expectativa de vida entre os sexos em 2010 para o estado de São Paulo);
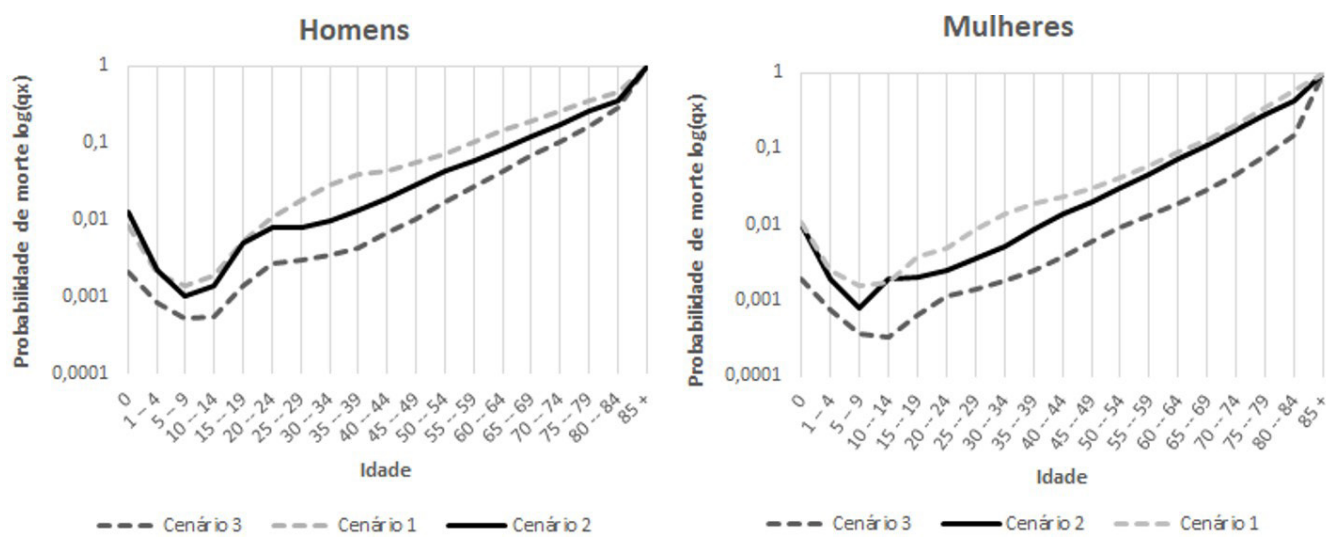

Figura 1. Probabilidade de morte no estado de São Paulo segundo a interpolação linear da função Logit de sobrevivência utilizando os diferentes cenários (2050). Fonte: elaborada a partir dos dados do SIM e HMD 
- Cenário 3.2: hipótese de ausência de gap entre os sexos (expectativa de vida das mulheres se iguala à dos homens);

- Cenário 3.3: hipótese de ausência gap entre os sexos (expectativa de vida dos homens se iguala à das mulheres)

\section{RESULTADOS}

\section{Projeção da população idosa do estado de São Paulo}

Após a realização da projeção via método estendido de coortes componentes, a projeção da população idosa de 65 anos ou mais e de 85 anos ou mais, segundo o cenário de projeção, é apresentado nas Figuras 2, 3, 4 e 5.

A projeção oficial da população idosa para o estado de São Paulo ${ }^{24}$ estima que $22,7 \%$ de pessoas tenham 65 anos ou mais em 2050. Em 2010, essa porcentagem representava 7,8\% da população total do estado. Usando a projeção realizada neste estudo, no cenário de alta mortalidade que considera uma maior mortalidade entre os adultos e maior mortalidade nas idades avançadas, a proporção de pessoas com 65 anos ou mais cairia para 20,2\% em 2050. Já em um padrão e nível de mortalidade parecido com o que ocorre com o Japão em 2010, a proporção de idosos com 65 anos ou mais seria de aproximadamente $26,3 \%$ da população total do estado em 2050. Mantendo as taxas constantes, o cenário é bem próximo ao projetado pela Fundação SEADE ${ }^{24}$, de 22,3\% das pessoas com 65 anos ou mais em 2050 (Figura 2). De acordo com essa variação de mortalidade, é possível ter uma diferença de 2,9 milhões de pessoas com essa faixa etária no estado, apenas considerando a variação do padrão e nível da mortalidade.

O impacto da variação da mortalidade considerando idosos mais velhos é ainda mais expressivo que nas idades mais jovens. Usando o cenário de alta mortalidade, estima-se que

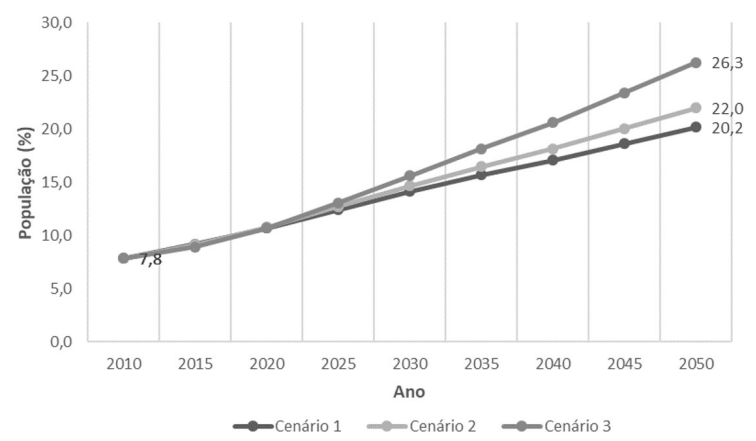

Figura 2. Projeção da proporção de pessoas com 65 anos ou mais, estado de São Paulo (2010-2050), cenários 1, 2 e 3. Fonte: elaborada por meio do software ProFamy

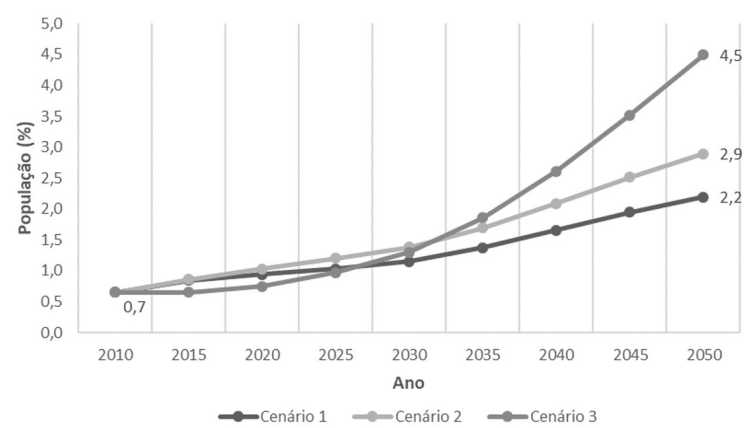

Figura 3. Projeção da proporção de pessoas com 85 anos ou mais, Estado de São Paulo (2010-2050), cenários 1, 2 e 3. Fonte: elaborada por meio do software ProFamy 


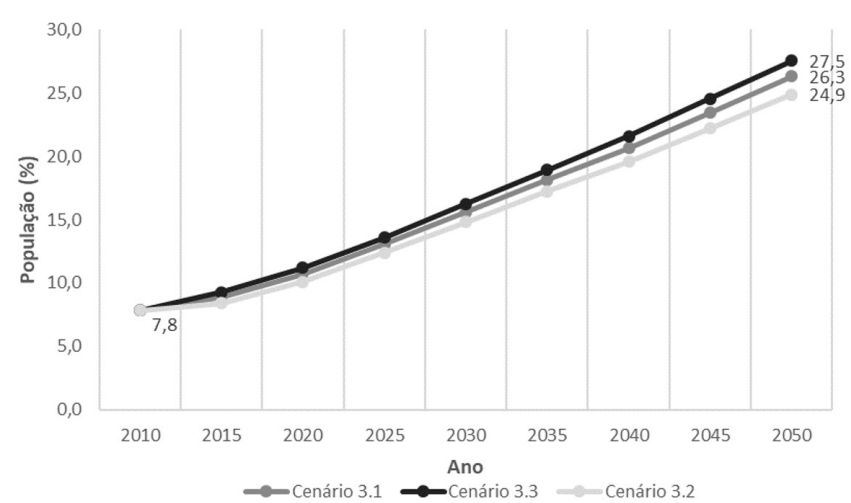

Figura 4. Projeção da proporção de pessoas com 65 anos ou mais, estado de São Paulo (2010-2050), cenários 3.1, 3.2 e 3.3. Fonte: elaborada por meio do software ProFamy

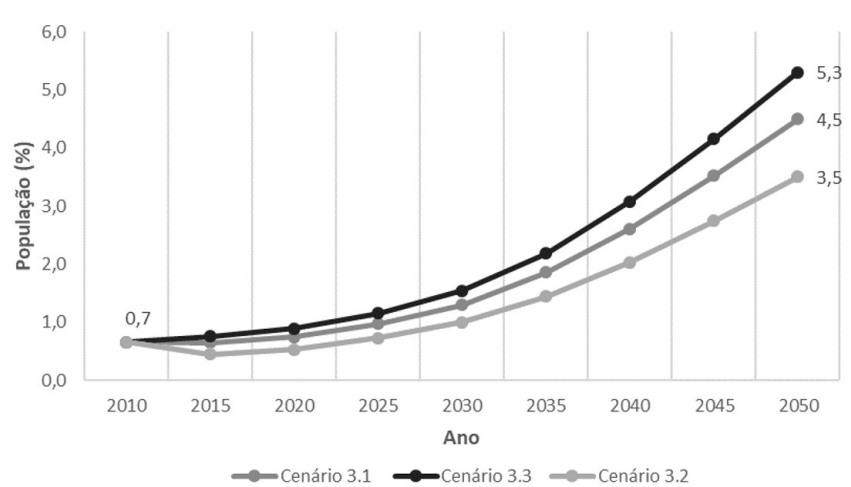

Figura 5. Projeção da proporção de pessoas com 85 anos ou mais, estado de São Paulo (2010-2050), cenários 3.1, 3.2 e 3.3. Fonte: elaborada por meio do software ProFamy

haja 2,2\% das pessoas com 85 anos ou mais em 2050 no estado. Usando o cenário de baixa mortalidade, essa porcentagem sobe para 4,5\%. Mantendo-se as taxas constantes, estimamse $2,9 \%$ das pessoas com 85 anos ou mais. De acordo com essa variação de mortalidade, é possível ter uma diferença de 1,1 milhão de pessoas com essa faixa etária no estado apenas considerando a variação do padrão e nível da mortalidade (Figura 3).

Dentro do mesmo cenário criado de baixa mortalidade, há uma variação significativa na projeção da população idosa se a diferença na expectativa de vida entre os sexos aumentar ou diminuir. Essa variação pode chegar a 2,6\% da população total, o que significa 1,2 milhão de pessoas no estado de São Paulo (Figura 4). A variação no gap entre os sexos tem um impacto muito maior na projeção populacional dos idosos com 80 anos ou mais. Nesse grupo, a projeção pode variar a 1,8\% da população total, ou seja, 0,8 milhão de pessoas no estado de São Paulo, assumindo um hipotético cenário de baixa mortalidade criado (Figura 5).

\section{DISCUSSÃO}

O objetivo deste trabalho não compreende fornecer estimativas da população idosa per se, dado que projeções populacionais são estudos muito mais complexos e demandam um número muito maior de variáveis para uma projeção dessa natureza. Entretanto, este trabalho representa um experimento importante para incorporar mudanças no comportamento da mortalidade em projeções de populações idosas.

Ao utilizar países em diferentes estágios da transição epidemiológica como cenário futuro do padrão e do nível da mortalidade de São Paulo, o método estendido de coortes componentes se torna uma possibilidade metodológica interessante para avaliar o impacto dessas modificações para a projeção da população idosa, podendo ser uma ferramenta para a 
avaliação de políticas públicas. Ainda assim, deve-se investir mais em pesquisas que utilizam métodos de projeção que permitam incorporar tanto as componentes demográficas quanto às variações na dinâmica populacional como ferramentas de análise.

\section{REFERÊNCIAS}

1. Omran AR. The epidemiological transition: a theory of the epidemiology of population change. Milbank Mem Fund Q. 1971;49(4):509-38. http://dx.doi.org/10.2307/3349375. PMid:5155251.

2. Horiuchi S. Healthy and mortality issues of global concern. In: Proceedings of the Symposium on Health and Mortality; 1997 nov 19-22; Brussels. USA: United Nations.

3. Frenk J, Frejka T, Bobadilla JL, Stern C, Lozano R, Sepúlveda J, et al. The epidemiologic transition in Latin America. Bol Oficina Sanit Panam. 1991;111(6):485-96. PMid:1838685.

4. Kannisto V, Lauritsen J, Thatcher AR, Vaupel JW. Reduction in Mortality at advanced ages: Several decades of evidence from 27 countries. Population and Denvelopment Review. 1994;20(4):793-810. http://dx.doi. org/10.2307/2137662.

5. Vaupel JW. How change in age-specific mortality affects life expectancy. Population Studies. 1986 mar;40(1):147-57. http://dx.doi.org/10.1080/0032472031000141896. PMid:11611920.

6. Guralnik JM, Yanagishita M, Schneider EL. Projecting the older population of the United States: Lessons from the past and prospects for the future. Milbank Q. 1988;66(2):283-308. http://dx.doi.org/10.2307/3350033. PMid:3185428.

7. Ahlburg DA, Vaupel JW. Alternative projections of the US population. Demography. 1990;27(4):639-52. http://dx.doi.org/10.2307/2061575. PMid:2249750.

8. Christensen K, Vaupel JW. Determinants of logevity: genetic, environmental and medical fator. J Intern Med. 1996;240(6):333-41. http://dx.doi.org/10.1046/j.1365-2796.1996.d01-2853.x. PMid:9010380.

9. Crimmins EM. Lifespan and healthspan: past, present, and promise. The Gerontologist. 2015;55(6):901-11 http://dx.doi.org/10.1093/geront/gnv130.

10. Passarino G, De Rango F, Montesanto A. Human longevity: genetics or Lifestyle? It takes two to tango. Immun Ageing. 2016;13:12. http://dx.doi.org/10.1186/s12979-016-0066-z. PMid:27053941.

11. Gonzaga MR. Medindo o processo de compressão da mortalidade no Brasil. Rev Bras Estud Popul. 2007 jul/dez;24(2):399-402.

12. Fries JF. The compression of morbidity. The Milbank Quarterly. 1983;61(3):397-419.

13. Wilmoth JR, Horiuchi S. Do the oldest old grow old more slowly? In: Robine JM, Forette B, Franceschi C, Allard M, editors. The paradoxes of longevity. Berlin, Heidelberg: Springer; 1999. p. 35-60. http://dx.doi. org/10.1007/978-3-642-60100-2_4.

14. Luy M, Miniguawa Y. Gender gaps—Life expectancy and proportion of life in poor health. Health Reports. 2014;25(12):12-9.

15. Luy M, Gast K. Do women live longer or do men die earlier? Reflections on the causes of sex differences in life expectancy. Gerontology. 2014;60(2):143-53. http://dx.doi.org/10.1159/000355310. PMid:24296637.

16. de Moura EC, Gomes R, Falcão MT, Schwarz E, das Neves AC, Santos W. Gender inequalities in external cause mortality in Brazil, 2010. Cien Saude Colet. 2015 mar;20(3):779-88. http://dx.doi.org/10.1590/141381232015203.11172014. PMid:25760118.

17. Zeng Y. Family dynamics in China: a life table analysis. Wisconsin: The University of Wisconsin; 1991.

18. Queiroz BL, Sawyer DOT. O que os dados de mortalidade do Censo de 2010 podem nos dizer? R Bras Est Pop. 2012;29(2):225-38.

19. Paes NA. Assessment of completeness of death reporting in Brazilian states for the year 2000. Rev Saude Publica. 2005;39(6):882-90. http://dx.doi.org/10.1590/S0034-89102005000600003. PMid:16341396.

20. Instituto Brasileiro de Geografia e Estatistica. REDE Interagencial de Informação para a Saúde - RIPSA Indicadores básicos para a saúde no Brasil: conceitos e aplicações - RIPSA. $2^{\text {a }}$ ed. Brasilia: Organização Pan-Americana de Saúde; 2008.

21. Zeng Y, Land KC, Gu D, Wang Z. Household and living arrangement projections: the extended cohortcomponent method and applications to the U.S. and China. New York, NY: Springer Netherlands; 2014. http://dx.doi.org/10.1007/978-90-481-8906-9.

22. Bongaarts J. The formal demography of families and households: an overview. Newsl Int Union Sci Study Popul. 1983;17:27-42. PMID: 12179533.

23. Centro Latino-Americano de Demografia. Métodos para proyecciones demográficas. San José, Costa Rica: CELADE; 1984.

24. SEADE. Sistema de Projeções Populacionais para os municípios do Estado de São Paulo. São Paulo, SP: SEADE; 2017.

25. Brasil. Ministério da Saúde. Banco de dados do Sistema Único de Saúde - DATASUS. Informações de Saúde, Sistema de Informações sobre Mortalidade. Disponível em http://tabnet.datasus.gov.br/cgi/idb2012/ a11 mb.htm [Citado 2020 Nov 3]. 